\title{
Mechanisms of selective toxicity of metronidazole and other nitroimidazole drugs
}

\author{
DAVID I EDWARDS \\ From the Chemotherapy Research Unit, Department of Paramedical Sciences, North-east London \\ Polytechnic, London
}

SUMMARY The selectively toxic effect of nitroimidazole drugs towards anaerobic bacteria and protozoa depends on a number of factors. The killing action of such drugs as metronidazole requires the reduction of the nitro group, a process which influences the rate of entry of the drug into the susceptible cell and which is determined by mechanisms involving ferredoxin-linked (or the equivalent) reactions in the cell. The reduced agent subsequently causes strand breakage of DNA, the extent of which depends on the A + T content of the DNA. Other effects of such drugs may include the possible inhibition of DNA repair mechanisms which exacerbate DNA damage. Inhibition of activity of nitroimidazoles may be caused by aminothiol radical scavengers and radioprotectors normally present in the cell or by the presence of other organisms in the environment (that is, the vagina) capable of inactivating the drugs.

\section{Introduction}

In 1978 , an editorial in this journal ${ }^{1}$ gave a short review of the types, availability, and relative activity of nitroimidazoles used primarily against Trichomonas vaginalis infections. It is now timely to review the mechanism of action of such drugs in an attempt to explain their selective action and their unique contribution to the chemotherapy of anaerobic organisms since their introduction in 1959.

The first of these drugs, metronidazole, has been studied more widely than any other and consequently will be used as the type example. Metronidazole (1- $\beta$-hydroxyethyl-2-methyl-5-nitroimidazole) is effective against a variety of protozoal diseases including trichomoniasis, ${ }^{2}$ giardiasis, ${ }^{3}$ amoebiasis, ${ }^{4}$ and balantidiasis, ${ }^{5}$ as well as being a valuable agent for the treatment of bacterial disease caused by anaerobes-for example, Vincent's disease ${ }^{6}$ and post-operative infections. ${ }^{7}{ }^{8}$ More recently, much interest has been shown in its possible application as a specific radiosensitiser of hypoxic tumours together with the 2-nitroimidazole, misonidazole. ${ }^{9-13}$

Other nitroimidazole drugs, such as tinidazole, sulnidazole, secnidazole, and ornidazole, have

Address for reprints: Dr D I Edwards, Chemotherapy Research Unit, Department of Paramedical Sciences, North-east London Polytechnic, Romford Road, London E15 4LZ

Received for publication 7 December 1979 broadly similar spectra of activity but none has sufficient advantages to supplant metronidazole from its role as the drug of choice for many bacterial and protozoal diseases. The structure of the drugs is shown in the figure. The spectrum of activity of nitroimidazoles is unique in that unlike any other antimicrobial drug they encompass Gram-positive and Gram-negative bacteria, protozoa, and even a few nematode worms, all of which are either anaerobic or micro-aerophilic. Thus the selective toxicity of these drugs rests on their ability to exert a cytotoxic effect specific to anaerobes.

\section{Mode of action}

The first studies on the mode of action of metronidazole indicated that it inhibited the output of $\mathrm{H}_{2}$ gas from $T$ vaginalis before that of $\mathrm{CO}_{2}$, which parallels cell death, indicating that $\mathrm{H}_{2}$ inhibition is a primary effect. ${ }^{14}$ Further studies showed that $\mathrm{H}_{2}$ output was by a phosphoroclastic mechanism of the clostridial type, in which pyruvate and phosphate are converted to acetylphosphate, $\mathrm{CO}_{2}$, and $\mathrm{H}_{2}$, and that nitroimidazoles act by accepting electrons from an electron transport protein which is normally involved in $\mathrm{H}_{2}$ gas evolution. ${ }^{1415}$ Since other drugs, for example, dimetridazole and tinidazole, have the same effect this inhibition appears to be a general property of 5-nitroimidazoles. However, the impaired function of the phosphoroclastic mechanism is not 

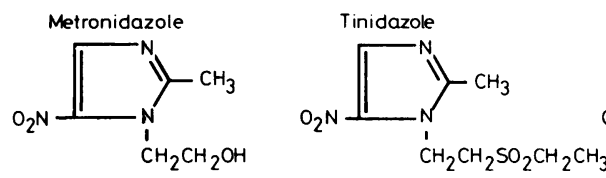

$4(5)$-Nitroimidazole

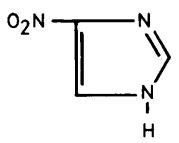

Misonidazole

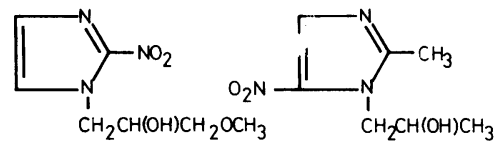

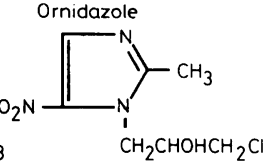

M \& B 4998

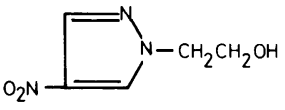

Sulnidazole

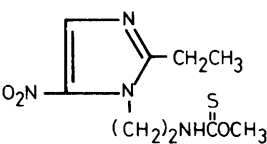

FIGURE Structures of nitroimidazole drugs. Metronidazole, tinidazole, ornidazole, secnidazole, and sulnidazole are 5-nitroimidazoles, and carnidazole is the 2-methyl congener of sulnidazole. Misonidazole is a 2-nitroimidazole, 8609 $R P$, a 4-nitroimidazole, and $M \& B$ 4998, a 4-nitropyrazole. responsible for death since the cell recovers once all the drug is reduced. ${ }^{16}$

Examination of the reduction process showed that the key step lies in the potential of the redox couple between the drug and the electron transfer protein, which in Clostridia is ferredoxin. The redox potential of such a reaction is about $-450 \mathrm{mV}$. These low potentials are not found in aerobes and consequently are one factor for the selective toxicity of nitroimidazoles towards anaerobes alone. It follows that any ferredoxin-linked system of the correct potential should be capable of reducing nitroimidazoles and causing cell death. This certainly occurs even with photosynthetic systems where it has been shown that metronidazole inhibits sugar synthesis in sugar cane leaves as a consequence of inhibiting photosystem 1 (where ferredoxin is involved) but has no effect on photosystem 2 , the Hill reaction. ${ }^{17}$ Further studies in spinach chloroplasts showed a direct inhibition of ferredoxinlinked nicotinamide adenine dinucleotide phosphate (NADP) reduction and corroborative studies have shown that although metronidazole has no effect on the chemo-organotrophic growth of Rhodopseudomonas acidophila in the dark, it kills it when grown photo-organotrophically in the light-that is, when ferredoxin-linked systems become operative. Only in ferredoxin-operative systems, or their equivalent, are nitroimidazoles reduced. ${ }^{15} 18$

These events in all susceptible cells have two important consequences. The first is that reduction of nitroimidazoles creates a favourable concentration gradient in which unreduced drug enters the cell relatively rapidly as the internal concentration is decreased due to reduction. This observation, first suggested by Ings et $a l,{ }^{19}$ implies that entry into aerobic cells is more difficult than into anaerobes and is consequently a further factor in explaining the selective toxicity of nitroimidazoles. The second is that reduction of the nitro group is a necessary function of the drugs' cytotoxic action.

The question remains however: if reduction of the drugs via the phosphoroclastic system is not the lethal event in the cell, what is? It becomes a logical hypothesis to propose that the biochemical lesion is caused not by the original drug molecule but by a reduction product or products which act at another site. The reduction of nitroimidazoles is merely an activation mechanism therefore which only anaerobes can carry out.

\section{Site of action}

Although protein has been suggested as a possible site of action of these drugs on the basis that about $30 \%$ of labelled metronidazole binds to intracellular components, the distribution of which parallels that of protein, ${ }^{20}$ no evidence has been published to suggest that any important protein system is inhibited. Instead, evidence has accumulated over the last few years since the suggestion of Ings et $a l^{19}$ that DNA is the major site of action of nitroimidazoles.

Ings' initial observations showed that DNA synthesis in $T$ vaginalis was inhibited as measured by incorporation of ${ }^{14} \mathrm{C}$-labelled thymidine into perchlorate-precipitable material in both the protozoan and in Clostridia. This work was subsequently corroborated and extended in our laboratory and showed that DNA synthesis was not only inhibited but existing DNA was broken down in Clostridia, and DNA could not be extracted from drug-treated Trichomonas or Clostridia, indicating 
that nitroimidazoles cause degradation of DNA. ${ }^{21}$ In this study it was apparent that metronidazole was a more potent degrader of DNA than tinidazole even though the latter acted sooner as a consequence of its ability to penetrate cells faster than metronidazole. Significant progress in the study of nitroimidazoles came in 1977, when it was found that chemically reduced metronidazole caused destabilisation of the DNA helix and strand breakage in vitro thus enabling model systems to be used. ${ }^{22}$ This latter work however could be criticised on the grounds that the reducing agent itself (dithionite) could cause DNA strand breakage thus making specific measurement of the drug's action difficult. ${ }^{23}$

To overcome the problems of chemical reduction of nitroimidazoles we have developed an electrolytic reduction technique which enables the effect of reduced nitroimidazole on DNA to be measured directly.

\section{Mechanism of action}

Polarography of nitroimidazoles enables an assessment of the redox potentials of the drugs to be measured. These potentials can then be used to select the voltage for drug reduction ensuring that it is relevant to those potentials found in anaerobic organisms. Previous studies have already shown that if DNA is added to previously reduced drug there is no effect, indicating that the reduced intermediate responsible for DNA damage is short-lived with a half-life of less than 1 second. If electrolytic reduction is carried out in the presence of DNA at voltages not exceeding minus $1 \cdot 1-1 \cdot 2 \mathrm{~V}$, which causes DNA denaturation, ${ }^{24} 25$ a number of techniques can be used to assess DNA damage. Generally, damage only occurs under anaerobic conditions-namely, an atmosphere of $\mathrm{N}_{2}$ - and as a consequence of the reduction of the nitro group of the drug, which can be monitored by measuring the decrease in the absorption maximum of each drug due to the nitro group. ${ }^{26}$

Recently, studies have been carried out on a variety of nitroimidazoles and nitropyrazoles and the damage to DNA characterised. The major techniques used in this type of study are viscometry of DNA solutions, measurements of the melting and renaturation profiles and its Tm value* using the Rowley equation, ${ }^{27}$ measurement of the single-strand content of DNA using hydroxyapatite chromatography, measurement of the decrease in molecular weight (and thus length) by acridine-impregnated agarose

*When DNA is heated it is converted from its normal doublestranded form (helix) to single strands (random coil) and this process is accompanied by an increase in absorbance. The midpoint of this helix-coil transition is known as the "melting" or Tm value and is expressed in degrees celsius. gel electrophoresis, and finally the assessment of single-and double-strand breaks in DNA by sucrose gradient sedimentation ultracentrifugation.

Such techniques have shown that reduced drugs decrease the viscosity of DNA (which is a general index of damage), decrease the $\mathrm{Tm}$, and increase the melting range (indicating a decrease in the stability of the molecule and an increase in its polydispersity) and inhibit renaturation (indicating loss of helix integrity). This loss of structural integrity of DNA may be attributed to a number of possible events, but the most obvious is that of scission of the phosphodiester backbone-that is, strand breakage. Confirmatory evidence for strand breakage comes from studies which show a drug-induced increased single-strand content of DNA, its decreased molecular length and weight, and direct measurement of single- and double-strand breaks by sucrose gradient sedimentation. ${ }^{27-30}$ These effects have been observed with metronidazole, misonidazole, 4-nitroimidazole, M \& B 4998 and 8609 RP (for structures see figure). The overall mechanism of action of all these drugs therefore is to decrease the stability and integrity of DNA by strand breakage.

Metronidazole and misonidazole are presently being evaluated as radio-sensitisers of hypoxic tumours, ${ }^{31}$ and the process of radiosensitisation has been extensively studied. This process has many features in common with the cytotoxic properties of nitroimidazoles, including the parameters of nitrogroup reduction and DNA strand breakage. Where the processes appear to differ is in the speed and temperature dependence. Thus radiosensitisation is a fast process (about 10-30 milliseconds) as is radiation damage (10 milliseconds) compared with radioprotection mechanisms (850 milliseconds) and cytotoxic effects (about 1 second) and is temperatureindependent, whereas cytotoxicity is relatively slow and temperature-dependent. It is now well established that the damaging agent of nitroimidazoles as radiosensitisers is the radical anion $\left(-\mathrm{NO}_{2}\right)$ since the efficiency of radiosensitisation of nitroimidazoles can be correlated with their oneelectron redox potentials. 3233

However, if the efficiency of strand-breakage of DNA in an electrolytic reduction in-vitro system is compared with the one-electron redox potential or the polarographic half-wave potential no obvious correlation exists ${ }^{27}$ indicating that the cytotoxic effect towards anaerobes is not due to the nitro radical anion. Recent observations in our laboratory ${ }^{30}$ show that reduced nitroimidazole-induced damage of DNA can be prevented by certain aminothiols, notably cysteamine and cystamine, and that cysteamine acts as a radical scavenger whereas cystamine does not but behaves as a radioprotector. 
This leads to the conclusion that the reduced agent responsible for DNA damage is at least further reduced beyond the radical anion stage. Precisely what this agent is has not been established yet because of its (their) inherent instability and short half-life. However, the implications of this cytotoxic agent are important for the anticancer action of the drugs since it has been established that the killing effect of the drugs in conjunction with radiation is greater than can be accounted for by radiosensitisation alone. The evidence for this comes from studies in which both normal (oxic) and hypoxic tumour cells are killed. ${ }^{34}$ Since the radiosensitisation effect occurs only in hypoxic cells the killing of oxic cells can only be accounted for by a cytotoxic mechanism which does not involve free radicals.

The model electrolytic reduction system is applicable therefore for the study of both the mechanisms of cytotoxicity in anaerobes and in hypoxic cells.

\section{Molecular basis of selective toxicity}

Studies of the relative damage done by a number of nitroimidazoles results in two important characteristics of drug action. The first is that the amount of damage varies with the base composition of DNA and the second is that the amount of protection afforded by cysteamine depends on the redox potential of the drug. This latter point is important since the efficiency of action on DNA depends on the degree of strand breakage and aminothiol protection within the cell.

As regards the base composition of DNAs, it can be shown that damage is directly proportional to the $A+T$ content irrespective of what technique is used to measure DNA damage (Edwards et al, unpublished observation) and that, using a variety of chromatographic techniques, it appears that thymidine phosphates are specifically released from DNA (Knox et al, unpublished observations). These results also argue against a free radical being responsible for such selective DNA damage. Precisely how specific thymidine phosphate release is achieved is not known and will require the elucidation of the active intermediate before this process is understood, but it does enable a molecular basis of selective toxicity to be formulated. Thus, one would expect nitroimidazoles to be particularly selectively toxic to anaerobic organisms which have $A+T$-rich DNAs and somewhat less so in G + Crich organisms. The protozoans, $T$ vaginalis and Entamoeba histolytica, have A + T contents of $71 \%$ and $62-78 \%$ respectively ${ }^{35-38}$ and Bacteroides have $A+T$ contents of about $59-61 \%$. These organisms are the main causes of disease for which nitroimidazole drugs have major clinical application. In contrast, Rhodospirillum and $R$ acidophila have relatively low $\mathrm{A}+\mathrm{T}$ contents (38\% and $33 \%$ respectively) and these organisms are about 25 times less susceptible to nitroimidazoles than the protozoa or bacteria based on relative MICs. The implications of this will be discussed in detail elsewhere, but it is obvious that the particular effectiveness of such $A+T$-specific drugs against $\mathrm{A}+\mathrm{T}$-rich DNA is highly germane to their spectrum of activity or taxonomic specificity. ${ }^{15}$

\section{Resistance and drug inactivation}

There have been many reports of trichomonads, including $T$ vaginalis, which are resistant to metronidazole or other nitroimidazoles or both. ${ }^{39-41}$ Most of these reports are either of no clinical significance, because resistance levels are well below therapeutically obtainable concentrations, or the result of non-anaerobic conditions. ${ }^{4041}$ Because the presence of $\mathrm{O}_{2}$ in cultures raises the redox potential to positive values drug reduction does not occur and MIC values will increase, erroneously indicating the presence of resistant organisms. The underlying biochemical mechanisms for this may involve carboxylation reactions in $T$ vaginalis. ${ }^{42}$ However, truly resistant organisms have been developed in the laboratory and these are cross-resistant to a wide variety of nitroimidazoles. ${ }^{39}$

It is unusual that no resistant organisms of clinical significance have arisen during 20 years' use with either metronidazole or any other nitroimidazoles. This would suggest that a single-gene change conferring resistance is itself lethal and indicative of a mechanism and site of action where gene mutation is not compatible with cell survival. However this does not necessarily mean that resistance to nitroimidazoles is impossible; indeed several reports indicate that resistance to nitroimidazoles is possible but not to an extent that would have any clinical significance. The major, albeit rare, occurrence is that of drug inactivation which manifests itself during treatment. In the case of $T$ vaginalis, the organism from refractory patients is invariably found to be sensitive in vitro. The reason for this is the presence of other organisms in the vagina, notably Streptococcus faecalis, Escherichia coli, Klebsiella, and Mimae, some of which can alone, or in combination, absorb sufficient amounts of drug to lower its effective concentration in the environment below that required for therapeutic purposes. Recent studies have shown that normally aerobic organisms are capable of absorbing amounts of metronidazole without their viability being significantly affected 
both in aerobic and anaerobic environments. ${ }^{43} 44$ This inactivation is a feature both of 5- and 2-nitroimidazoles and may well be relevant to inactivation in other clinically relevant situations, for example, anaerobes in pus.

Clearly, because of the molecular basis of action of nitroimidazoles, resistance could not occur by way of an altered DNA or enzymes involved in DNA biosynthesis. Theoretically however there are mechanisms by which resistance to nitroimidazoles could be accomplished. Since entry into the cell is a prerequisite for drug activation (by reduction) an alteration in membrane permeability to exclude nitroimidazoles could lead to resistance. Attractive though this mechanism may be it must be remembered that nitroimidazoles are small molecules which enter the cell passively and there is no competition for uptake by other imidazoles, for example, histidine, histamine (Edwards, unpublished observations). Any alteration in membrane permeability to exclude nitroimidazoles could well also prevent uptake of histamine or histidine and other small molecules and be lethal to the cell.

A more logical mechanism of resistance would be based not on entry to the susceptible cell but on nonactivation of the drug, that is, no reduction of the nitro group. Since the mechanism for reduction lies in the pyruvate phosphoroclastic reaction one would expect a mutation in one of the enzymes of this complex such that the nitroimidazole is not accepted as a substrate. It must be pointed out that resistance to nitrofurans occurs by way of an altered nitroreductase enzyme and that these drugs are not reduced via a ferredoxin-linked mechanism because their redox potential is too positive. ${ }^{45} 46$

It is of great interest that Britz and Wilkinson ${ }^{47}$ have recently produced metronidazole resistance in Bacteroides using powerful mutagens, but the treated organisms have very much reduced levels of pyruvate dehydrogenase, a key enzyme involved in the phosphoroclastic reaction. Although no resistance mechanisms of this type in protozoa have been reported there have been reports of metronidazoleresistant Bacteroides resulting from long-term therapy, ${ }^{48}$ and it would be interesting to discover the precise mechanism of resistance of those organisms which are resistant to clinically significant concentrations of drug.

My thanks are due to the Medical Research Council, the Science Research Council, and the Cancer Research Campaign for financial support.

\section{References}

1. Anonymous. The nitroimidazole family of drugs. $\mathrm{Br} J$ Vener Dis 1978;54:69-71.
2. Cosar C, Julon L. Activité de l'(hydroxy-2-ethyl)-1-methyl-2nitro-5-imidazole (8823 RP) vis-à-vis des infections experimentales à Trichomonas vaginalis. Ann Inst Pasteur 1959;96:238-41.

3. Powell SJ, McLeod I, Wilmott AJ, Elsdon-Drew R. Metronidazole in amoebic dysentery and hepatic liver abscess. Lancet 1966; ii: 1329-31.

4. Khambatta RB. Metronidazole in giardiasis. Ann Trop Med Parasitol 1971;65:487-9.

5. Garcia-Leverde A, de Bonilla L. Clinical trials with metronidazole in human balantidiasis. Am J Trop Med Hyg 1975;24:781-3.

6. Davies AH, McFadzean JA, Squires S. Treatment of Vincent's stomatitis with metronidazole. $\mathrm{Br}$ Med $J$ 1964; i: 1149-50.

7. Willis AT, Ferguson IR, Jones PH, et al. Metronidazole in the prevention and treatment of Bacteroides infections in gynaecological patients. Lancet 1974; i: 1540.

8. Willis AT, Ferguson IR, Jones PH, et al. An evaluation of metronidazole in the prophylaxis and treatment of anaerobic infections in surgical patients. J Antimicrob Chemother $1975 ; 1: 393-401$.

9. Foster JL, Willson RL. Radiosensitization of anoxic cells by metronidazole. Br J Radiol 1973;46:234-5.

10. Deutsch G, Foster JL, McFadzean JA, Parnell M. Human studies with "high dose" metronidazole, a non-toxic radiosensitizer of hypoxic cells. $\mathrm{Br} J$ Cancer 1975; 31:75-80.

11. Urtasun RC, Chapman JD, Band P, Rabin H, Fryer C, Sturmwind J. Phase 1 study of high dose metronidazole. An invivo and in-vitro specific radiosensitizer in hypoxic cells. Radiology 1975; 117: 129-33.

12. Urtasun RC, Band P, Chapman JD, Feldstein ML, Mielke B Fryer C. Radiation and high dose metronidazole in supratentorial glioblastomas. N Engl J Med 1976; 284: 1357-64.

13. Dische S, Adams GE. Use of a hypoxic cell sensitizer misonidazole to improve radiotherapy. In: Siegenthaler W, Luthy R, eds. Current Chemotherapy. Washington: American Society for Microbiology, 1978: 1191-2.

14. Edwards DI, Mathison GE. The mode of action of metronidazole against Trichomonas vaginalis. J Gen Microbiol 1970;63:297-302.

15. Edwards DI, Dye M, Carne $H$. The selective toxicity of antimicrobial nitroheterocyclic drugs. J Gen Microbiol 1973; 76: 135-45.

16. O'Brien RW, Morris JG. Effect of metronidazole on hydrogen production in Clostridium acetobutylicum. Arch Microbiol $1974 ; 84: 225-33$

17. Edwards DI, Schoolar AI. Inhibition of sugar synthesis and potentiation of chlorophyll degradation in sugar cane leaf tissue by metronidazole. Z Pflanzenphysiol 1971;64:73-4.

18. Edwards DI, Mathison GE, Platt DJ. Metronidazole-an antimicrobial drug which inhibits photosynthesis. $Z$ Pflanzenphysiol 1974;71:424-7.

19. Ings RMJ, McFadzean JA, Ormerod WE. The mode of action of metronidazole in Trichomonas vaginalis and other organisms. Biochem Pharmacol 1974; 15 : 1421-9.

20. Muller M, Lindmark DG, McLaughlin J. Mode of action of metronidazole on anaerobic micro-organisms. In: Finegold SM, ed. Metronidazole. Amsterdam: Excerpta Medica 1977: 12-9.

21. Plant CW, Edwards DI. The effect of tinidazole, metronidazole and nitrofurazone on nucleic acid synthesis in Clostridium bifermentans. J Antimicrob Chemother 1976; 2:203-9.

22. Edwards DI. The action of metronidazole on DNA. $J$ Antimicrob Chemother 1977;3:43-8.

23. Cone R, Hasan SK, Lown JW, Morgan AR. The mechanism of degradation of DNA by streptonigrin. Can $j$ Biochem $1976 ; 54: 219-23$

24. Brabek, V, Palecek EC. Interaction of nucleic acids with electrically charged surfaces. III Surface denaturation of DNA on the mercury electrode in two potential regions. Stud Biophys 1976; 60: 105-10.

25. Nurnberg HW, Valenta P. Bioelectrical behaviour and deconformation of native DNA at charged interfaces. Colston Papers 1978; 29:201-29.

26. Edwards DI, Knight RC, Kantor I. Interaction of nitroimidazole drugs with DNA. In: Siegenthaler W, Luthy R, eds. Current Chemotherapy. Washington: American Society for Microbiology, 1978:714-6. 
27. Rowley DR, Knight RC, Skolimowski IM, Edwards DI. The effect of nitroheterocyclic drugs on DNA: an in-vitro model of cytotoxicity. Biochem Pharmacol 1979; 28: 3009-13.

28. Knight RC, Skolimowski IM, Edwards DI. The interaction of reduced metronidazole with DNA. Biochem Pharmacol 1978; 27:2089-93.

29. Knight RC, Rowley DA, Skolimowski IM, Edwards DI Mechanism of action of nitroimidazole antimicrobial and antitumour radiosensitizing drugs: effects of reduced misonidazole on DNA. Int J Radiat Biol 1979;36:367-77.

30. Edwards DI, Rowley DA, Knox RJ, Skolimowski IM, Knight RC. The nature of DNA damage induced by electrolytically reduced nitroimidazole drugs. In: Nelson JD, Grassi C, eds. Current Chemotherapy and Infectious Disease. Washington: American Society for Microbiology, 1980: 561-3.

31. Breccia A, Rimondi C, Adams GE. Radiosensitizers of Hypoxic Cells. Amsterdam: Elsevier (North Holland), 1979.

32. Wardman P. The use of nitroaromatic compounds as hypoxic cell radiosensitizers. Curr Top Radiat Res Quart 1977; 11:347-98.

33. Adams GE, Flockhart IR, Smithen CE, Stratford IJ, Wardman P, Watts ME. Electron-affinic sensitization. VII A correlation between structures, one-electron redox potentials and efficiences of nitroimidazoles as hypoxic cell radiosensitizers. Radiat Res 1978;67:9-20.

34. Brown JM, Yu NY, Cory MJ, Bicknell RB, Taylor DL. In-vivo evaluation of the radiosensitizing and cytotoxic properties of newly synthesized electron-affinic drugs. Br J Cancer 1978;37, suppl 3:206-11.

35. Mandel $\mathbf{M}$, Honigberg $\mathbf{B M}$. Isolation and characterization of DNA of two species of Trichomonas Donné. J Protozool 1964;11:114-6.

36. Gelderman AH, Keister DB, Bartgis L, Diamond LS. Characterization of the deoxyribonucleic acid of representative strains of Entamoeba histolytica, E histolytica-like amoeba and E moshkovskii. J Parasitol 1971;57:906-11.
37. Gelderman AH, Bartgis L. Keister DB, Diamond LS A comparison of the genome size and thermal denaturationderived base composition of DNAs from several members of Entamoeba (histolytica group). J Parasitol 1971;57:912-6.

38. Reeves RE, Lushbaugh $B$, Montalbo FE. Characterization of deoxyribonucleic acid of Entamoeba histolytica by caesium chloride density centrifugation. $J$ Parasitol 1971;57:939-44.

39. Meingassner JG, Mieth $\mathrm{H}$. Cross-resistence of trichomonads to 5-nitroimidazole derivatives. Experientia 1976;32:183-4.

40. Meingassner JG, Mieth H, Czok R, Lindmark DG, Muller M Assay conditions and the demonstration of nitroimidazole resistance in Tritrichomonas foetus. Antimicrob Agents Chemother 1978; 13: 1-3.

41. Meingassner JG, Thurner J. Strain of Trichomonas vaginalis resistant to metronidazole and other 5-nitroimidazoles. Antimicrob Agents Chemother 1979; 15:254-7.

42. Mack S, Muller M. Effect of oxygen and carbon dioxide on the growth of Trichomonas vaginalis and Tritrichomonas foetus. J Parasitol 1978;64:927-9.

43. Ralph ED, Clarke DA, Inactivation of metronidazole by anaerobic and aerobic bacteria. Antimicrob Agents Chemother $1978 ; 14: 377-83$.

44. Edwards DI, Thompson EJ, Tomusange J, Shanson D. Inactivation of metronidazole by aerobic organisms. $J$ Antimicrob Chemother 1979;5:315-6.

45. McCalla DR. Biological effects of nitrofurans. J Antimicrob Chemother 1977; 3:517-20.

46. McCalla DR. Nitrofurans. In: Hahn O, ed. Antibiotics. Vol 5. New York: Springer-Verlag, 1980: 176-213.

47. Britz ML, Wilkinson RG. Isolation and properties of metronidazole-resistant mutant of Bacteroides fragilis. Antimicrob Agents Chemother 1979; 16:19-27.

48. Ingham HR, Eaton S, Venables CW, Adams PC. Bacteroides fragilis resistant to metronidazole after long-term therapy. Lancet 1978; i: 214. 